\title{
Immunobiology of Ebola and Lassa virus infections
}

\author{
Joseph B. Prescott ${ }^{1}$, Andrea Marzi ${ }^{1}$, David Safronetz ${ }^{2,3}$, Shelly J. Robertson ${ }^{1}$, \\ Heinz Feldmann ${ }^{1,3}$ and Sonja M. Best ${ }^{1}$
}

Abstract | Two of the most important contemporary emerging viruses that affect human health in Africa are Ebola virus (EBOV) and Lassa virus (LASV). The 2013-2016 West African outbreak of EBOV was responsible for more than 11,000 deaths, primarily in Guinea, Sierra Leone and Liberia. LASV is constantly emerging in these and surrounding West African countries, with an estimate of more than 500,000 cases of Lassa fever, and approximately 5,000 deaths, annually. Both EBOV and LASV are zoonotic, and human infection often results in a severe haemorrhagic fever in both cases. However, the contribution of specific immune responses to disease differs between EBOV and LASV. This Review examines innate and adaptive immune responses to these viruses with the goal of delineating responses that are associated with protective versus pathogenic outcomes.

Haemorrhagic fever A fever and bleeding disorder that can progress to shock and death in many cases.
'Laboratory of Virology, Rocky Mountain Laboratories, National Institute of Allergy and Infectious Diseases, National Institutes of Health, 903S. Fourth Street, Hamilton, Montana 59840 USA.

2Zoonotic Diseases and Special Pathogens, Public Health Agency of Canada, 1015 Arlington Street, Winnipeg, Manitoba R3E 3R2, Canada. ${ }^{3}$ Department of Medical Microbiology, University of Manitoba, 745 Bannatyne Avenue, Winnipeg, Manitoba R3E OJ9, Canada.

Correspondence to H.F and S.M.B.

feldmannh@niaid.nih.gov; sbest@niaid.nih.gov
Ebola virus (EBOV; Zaire ebolavirus) and Lassa virus (LASV) belong to two separate virus families, the Filoviridae and Arenaviridae, respectively, but they share several biological characteristics. They are both emerging zoonotic viruses in central Africa that cause severe haemorrhagic fever in humans. Their threat to public health is in part due to their high virulence and their potential for human-to-human transmission. EBOV and LASV also have similarities in their pathogenesis in terms of their initial target cells being myeloid cells and in terms of the organs that are affected. However, the specific immune responses that are induced by infection and their roles in protection or pathogenesis are distinct. These differences may have important implications for understanding disease and therefore for the treatment of patients with haemorrhagic fever. This Review collates the current understanding of immunity to EBOV and LASV to illustrate differences between the two viral haemorrhagic syndromes. This information may also be relevant when considering the underlying pathogenesis of other severe haemorrhagic fevers caused by viruses, such as dengue virus or yellow fever virus (reviewed in REF. 1). A detailed knowledge of natural immunity to these emerging viruses may direct the development of therapies that augment protective immune responses.

EBOV is the aetiological agent of Ebola haemorrhagic fever, now known as Ebola virus disease (EVD) in humans ${ }^{2,3}$ (BOX 1). It was discovered in 1976 during an outbreak in northern Zaire (now Democratic Republic of the Congo (DRC)). Since then, multiple smaller outbreaks of EBOV have occurred in western Central African countries such as Gabon, DRC and Republic of the Congo ${ }^{3}$. More recently, EBOV emerged in West Africa, causing an unprecedented epidemic of approximately 30,000 cases, with effects on regional and global public health ${ }^{2}$ (FIG. 1). In addition, other Ebolavirus species have emerged as human pathogens in eastern Central Africa (Sudan ebolavirus and Bundibugyo ebolavirus) and in West Africa (Tai Forest ebolavirus). A fifth species of Ebolavirus, which is probably apathogenic for humans, emerged in the Philippines (Reston ebolavirus $)^{3,4}$. Marburg virus, a closely related member of the Filoviridae, causes a disease in humans similar to that of EBOV, and is responsible for periodic outbreaks in sub-Saharan Africa. The animal reservoirs of Ebolavirus remain unidentified, but they are likely to be found among fruit bats. Person-to-person transmission of Ebolavirus, which is a feature of human outbreaks, occurs largely through interaction with infected individuals involving direct contact with infectious body fluids. Other animal species such as the great apes can also develop Ebola haemorrhagic fever and are a source for human infection. At the present time, Ebolavirus is a sporadic zoonosis in equatorial Africa and now, perhaps, in West Africa, but there is no convincing evidence that EBOV is endemic in any area.

LASV is the aetiological agent of Lassa fever, which was first documented in 1969 during an outbreak on the Jos plateau in Nigeria ${ }^{5}$ (BOX 1). It is now known that Lassa fever is an endemic zoonosis in parts of West Africa, specifically Nigeria, Liberia, Sierra Leone and Guinea. Recent studies have also shown the presence of LASV in surrounding countries including Mali, Ivory Coast, Benin and Ghana, which indicates that the geographic 


\section{Zoonosis}

Any disease or infection that is naturally transmissible from vertebrate animals to humans.

Cytokine storm

A positive feedback loop involving the hypersecretion of cytokines with detrimental effects region of the virus and potentially of human disease may be expanding 6,7 (FIG. 1). Throughout West Africa, up to 500,000 people are infected annually with LASV, resulting in approximately 5,000 deaths ${ }^{8}$. In addition, a newly recognized Old World relative of LASV, called Lujo virus, was identified in 2008 as the causative agent of viral haemorrhagic fever in Zambia ${ }^{9}$. Most commonly, contact with infected rodent hosts (Mastomys natalensis; the common African rat) or ingestion and/or inhalation of virus-laden particles is the source of human LASV infection. However, person-to-person transmission can occur in hospital settings ${ }^{10}$.

Both EBOV and LASV cause haemorrhagic fever, albeit with differing pathophysiology. These viruses share several features, including their targeting of myeloid cells (macrophages and dendritic cells (DCs)), their ability to antagonize the innate immune system, and their ability to disrupt the functions of the adaptive immune response. Most of what is known regarding immune responses to infection with EBOV or LASV has been determined in animal models of infection and/or disease ${ }^{11,12}$, or from the in vitro culture of human cells. Animal models for EBOV include laboratory mice (sometimes using immunocompromised mice), humanized mice, guinea pigs, hamsters, ferrets and non-human primates; however, the virus requires adaptation to cause disease in immunocompetent rodent models. Certain guinea pig strains and non-human primates are useful models for LASV. Although much work has been carried out studying EBOV in mice, mice are resistant to disease in the absence of virus adaption, infection of mice

\section{Box 1 | Basic virology of EBOV and LASV}

Ebola virus (EBOV) is the prototypical member of the Filoviridae family; it is taxonomically classified in the genus Ebolavirus, species Zaire ebolavirus ${ }^{4}$. Virions of EBOV are enveloped, having a diameter of approximately $80 \mathrm{~nm}$ but being variable in length and shape. The genome consists of non-segmented, negative-sense RNA of $\sim 19 \mathrm{~kb}$ with the following gene order: $3^{\prime}$ leader, nucleoprotein (NP), virion protein 35 (VP35), VP40, glycoprotein (GP), VP30, VP24, polymerase (L) and 5' trailer. Transcription and replication occur in the cytoplasm. Promoter and packaging signals are located in the conserved leader and trailer regions. Each gene is flanked by conserved transcriptional start and stop signals, and most genes are separated by intergenic regions. The minimal functional complex for transcription and replication consists of NP, VP35, VP30, L and genomic RNA. VP40 mediates particle formation and connects the nucleocapsid to the plasma membrane at sites where GP is inserted before virion budding. The GP gene also encodes two soluble glycoproteins (sGP and ssGP), of which sGP may function as an antibody decoy. VP35 and VP24 have strong interferon antagonistic properties and are important viral pathogenicity factors 3 330,127,128.

Lassa virus (LASV) is the prototypical member of the Arenaviridae family, genus Mammarenavirus ${ }^{23}$. Enveloped virions of LASV are pleomorphic, with diameters ranging from $50 \mathrm{~nm}$ to $300 \mathrm{~nm}$, and contain a single-stranded, bi-segmented, ambisense RNA genome of $\sim 10.5 \mathrm{~kb}$. Each genetic segment encodes two non-overlapping open reading frames of opposite polarity that are separated by a short hairpin region. The large segment encodes the viral polymerase $(\mathrm{L})$ and zinc-binding matrix protein $(\mathrm{Z})$; the small segment encodes NP and GPs, G1 and G2. The $5^{\prime}$ and $3^{\prime}$ ends of both segments contain conserved complementary nucleotides, resulting in the appearance of circular genomic segments. Viral transcription and replication take place in the cytoplasm. NP and $L$ are transcribed from genomic RNA, whereas $G 1, G 2$ and $Z$ are transcribed only from antisense transcripts of the genome. The intergenic stem-loop hairpins on both segments function as transcription terminators. Z-mediated budding occurs at the plasma membrane where $\mathrm{G} 1$ and $\mathrm{G} 2$ are inserted. LASV particles also contain host ribosomes, which gives the virus its characteristic grainy morphology. rarely recapitulates the hallmarks of human disease, and immune responses can differ between rodents and primates $^{13}$. Therefore, this Review focuses on information derived from studies with humans and non-human primates. We discuss immune responses in survivors of infection compared with in individuals who succumb to infection to highlight the features of a protective immune response. However, some aspects of immunity cannot be readily studied in humans or non-human primate models and, therefore, the information given is supplemented by observations from rodents where necessary.

\section{Disease course and treatment}

EVD. EBOV infections are severe in nature and associated with case fatality rates ranging from $45 \%$ to $90 \%$. The incubation period is up to 21 days with abrupt onset of fever concurrent with malaise, fatigue, myalgia and headache. Subsequently, patients may develop nausea, vomiting, profuse watery diarrhoea, haemorrhages, meningoencephalitis, and hepatic and renal injury. Infection leads to systemic viral replication, target cell necrosis and the apoptosis of bystander lymphocytes, and it induces a cytokine storm that results in hypovolaemic shock and multi-organ failure ${ }^{3,14,15}$. If the infection is survived, recovery generally occurs after $2-3$ weeks, but convalescence is associated with sequelae, known as post-Ebola syndrome, that may include fatigue, uveitis, hearing loss, arthralgia and anxiety ${ }^{16,17}$. EVD causes high rates of maternal and perinatal mortality, with most cases of infection during pregnancy ending in spontaneous miscarriage, stillbirth or neonatal death. EBOV crosses the placenta to infect the fetus and the amniotic fluid ${ }^{18,19}$.

Lassa fever. The majority of LASV infections are asymptomatic or mild in nature $\mathrm{e}^{20-22}$. It is generally thought that approximately $20 \%$ of infections result in moderate to severe disease, which can be associated with haemorrhagic manifestations and multi-organ failure $e^{8,23}$. The incubation period is up to 21 days with a gradual onset of fever, headache, myalgia and arthralgia, as well as pharyngitis with a non-productive cough. Vomiting, diarrhoea, increased levels of liver enzymes (such as aspartate transaminase (AST) and alanine transaminase $(\mathrm{ALT}))$ in the blood and a raised haematocrit are often observed in severe cases of Lassa fever. Abdominal and retrosternal pain, oedema of the face and neck, enlarged lymph nodes and/or haemorrhage in the conjunctiva or mucosal surfaces are particularly indicative of a poor prognosis. Recovery generally takes $1-3$ weeks and can be associated with hearing loss. LASV infection in pregnancy, especially during the third trimester, has particularly severe effects, with the maternal mortality rate estimated at $20 \%$ and the fetal mortality rate being near $100 \%$. In children, infection is associated with a 'swollen baby' syndrome consisting of widespread oedema, abdominal distension and bleeding ${ }^{8,23}$.

Therapies. The wide range of clinical presentations and the lack of distinguishing symptoms early in infection hamper the clinical diagnosis, even by experienced physicians, of EVD, Lassa fever and other tropical diseases, 

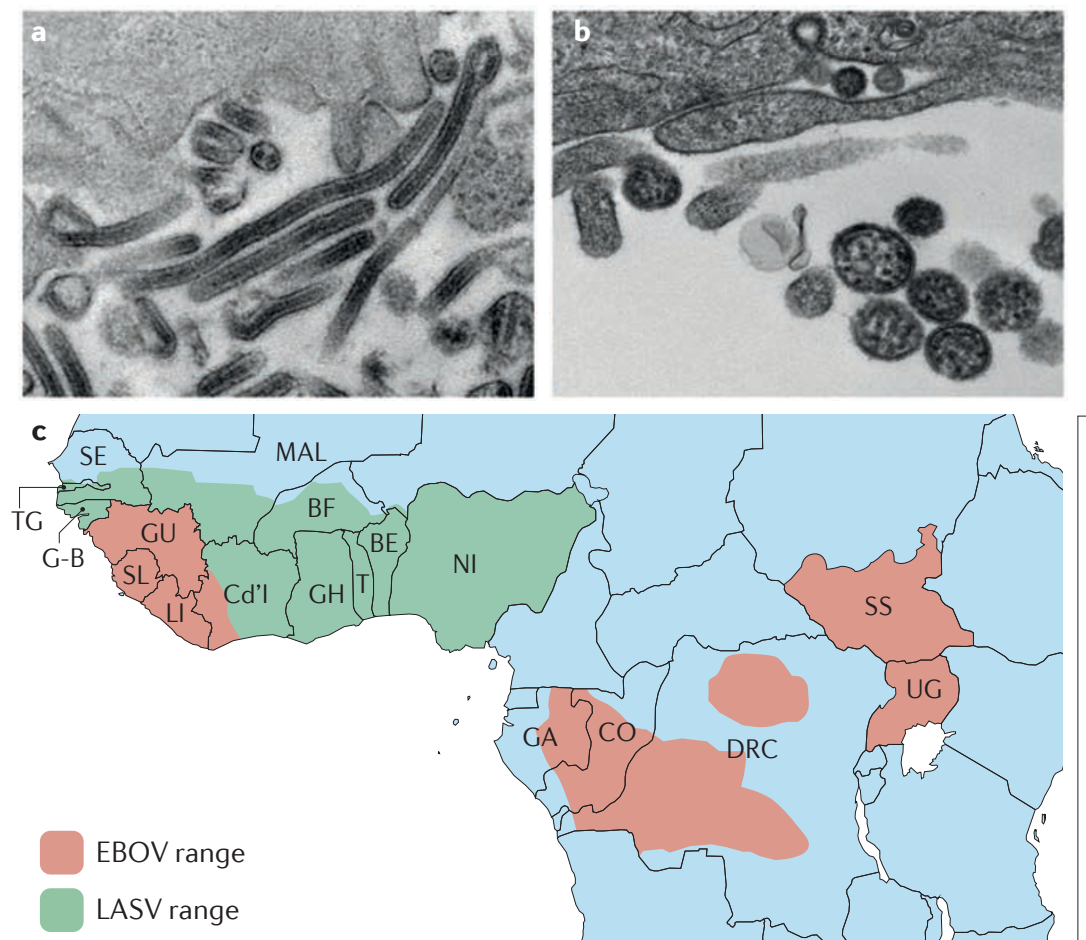

\begin{tabular}{l} 
SE - Senegal \\
TG - The Gambia \\
G-B - Guinea-Bissau \\
MAL - Mali \\
BF - Burkina Faso \\
GU - Guinea \\
SL - Sierra Leone \\
LI - Liberia \\
Cd'I - Cote d'lvoire \\
GH - Ghana \\
T $~-~ T o g o$ \\
BE - Benin \\
NI - Nigeria \\
SS - South Sudan \\
UG - Uganda \\
GA - Gabon \\
CO - Republic of the Congo \\
DRC - Democratic Republic \\
$\quad$ of the Congo \\
\hline
\end{tabular}

Figure 1 | Visualization of EBOV and LASV and their distribution in Africa. The morphology of Ebola virus (EBOV; part a) and Lassa virus (LASV; part b) particles is shown by transmission electron microscopy of Vero E6-infected cells. The filamentous morphology and hallmark shepherd's crook are visible for EBOV. The granular appearance resulting from host cell-derived ribosomes can be seen for LASV, which gives the viruses of the family Arenaviridae its name (arena is Latin for sand). The map shows the distribution of these two viruses in Africa, with LASV continuously emerging in West Africa, and EBOV sporadically emerging as a zoonotic infection in Central and recently also West Africa (part c). Images in part a and part b provided by Elizabeth Fischer, National Institute of Allergy and Infectious Diseases, National Institutes of Health, USA.

such as yellow fever, dengue fever, Crimean Congo haemorrhagic fever, malaria and leptospirosis. Currently, there are no licensed treatments specific for either EVD or Lassa fever. The broad-spectrum antiviral ribavirin seems to benefit patients with Lassa fever if the drug is administered early in the course of disease. Although patients with EVD may benefit from treatment with antibody cocktails $^{24}$ and with certain other drugs such as the antiviral favipiravir ${ }^{25}$, the most beneficial current treatment for patients with EVD or Lassa fever is aggressive supportive care. Therefore, rapid and reliable diagnosis of EBOV and LASV infection is essential to reduce morbidity and mortality and to support public health measures, such as patient isolation and contact tracing. There are no licensed vaccines to prevent EBOV and LASV infections. However, experimental approaches have shown promising preclinical results, and ring vaccination under an investigational protocol using a vesicular stomatitis virus (VSV)-based EBOV vaccine has demonstrated efficacy against EBOV in the recent West African outbreak ${ }^{26}$ (BOX 2).

Ring vaccination A vaccination strategy whereby the most likely contacts of an infected person are vaccinated in an attempt to control an outbreak of a highly transmissible disease.
Early events that shape the immune response. Both EBOV and LASV initially target myeloid cells, specifically myeloid DCs (mDCs), monocytes and macrophages, although the particular type of initial target cell may vary according to the specific route of infection.

\section{Innate immunity to EBOV and LASV}

Stromal DCs or Langerhans cells of the epithelium, or mucosal DCs or macrophages are most likely the first cells to be infected ${ }^{27-29}$. These cell types support high levels of viral replication, although additional cell types such as endothelial or parenchymal cells cannot be ruled out as early targets. Virus replication in DCs and macrophages is associated with disruption of cellular function in terms of antigen presentation and the induction of an adaptive immune response, thus initiating the immune dysregulation that is observed in patients.

For EBOV, at least in non-human primates, infected $\mathrm{mDCs}$ home to the draining lymph nodes, carrying the virus with them ${ }^{27}$. Macrophages, but not DCs, are activated by infection and produce large amounts of inflammatory cytokines. The importance of early events in these cells in determining the outcome of infection may be best illustrated by comparing the replication of adapted and non-adapted viruses in non-permissive hosts. Infection of rodents (mice and hamsters) with EBOV does not cause disease unless the virus has been previously adapted to replication in mice ${ }^{30-32}$. Comparing infection with EBOV and with mouse-adapted (MA)EBOV in hamsters suggests that replication of the nonadapted virus is restricted to myeloid cells followed by virus clearance, whereas MA-EBOV progresses beyond myeloid cells in the liver to infect hepatocytes ${ }^{33,34}$. Therefore, myeloid cells are an important barrier to viral 
dissemination, and the ability of EBOV to manipulate this specific cellular environment is probably crucial in determining the control of virus replication and disease.

Similar to EBOV, the primary target cells of LASV are cells of the myeloid lineage, including macrophages and $\mathrm{DCs}^{35,36}$; however, in contrast to EBOV, replication of LASV fails to lead to the activation or maturation of either cell type. To evaluate the quality and extent of myeloid cell dysregulation in LASV infection, non-pathogenic arenaviruses have been directly compared with LASV for their ability to activate these cell types in vitro. Mopeia virus (MOPV) is vectored by M. natalensis as is LASV, and MOPV has high levels of amino acid similarity with LASV; however, MOPV is apathogenic in humans and non-human primates ${ }^{37,38}$. Following infection with MOPV, DCs and macrophages support high levels of viral replication, which is similar to LASV; in contrast to LASV, DCs and macrophages are strongly activated by MOPV infection and increase their expression levels of CD80, CD86 and CD40, as well as the expression of type I interferons (IFNs), tumour necrosis factor and interleukin-6 (IL-6) ${ }^{35}$. These data indicate that high levels of activation of myeloid cells by MOPV may be a feature of a protective immune response and that the failure of LASV to activate myeloid cells, or its ability to directly antagonize the activation of these cells, is a determinant of pathogenesis. Furthermore, the lack of immune activation in response to LASV, resulting from inhibited DC maturation, may be accompanied by immune tolerance to LASV, which can be induced by the presentation of viral antigens by immature DCs, leading to active immunosuppression ${ }^{39}$.

Both EBOV and LASV arrest DCs in an immature, inactive state despite DC migration to the draining lymph nodes ${ }^{40}$, whereas macrophages are activated by
$\mathrm{EBOV}^{41}$ but not by LASV. The more complete disruption of the function of antigen-presenting cells (APCs) by LASV compared with EBOV might correlate with the general lack of adaptive immune responses in fatal Lassa fever (see below), whereas the ability of at least macrophages to function as effective APCs early in the immune response to EBOV might enable the strong and early activation of the adaptive immune response seen in fatal EVD. It is important to note that almost all observations regarding interactions between these viruses and myeloid cells have been made using human cells isolated and derived in cell culture, which probably does not accurately reflect all features of infection and cell maturation in vivo. Nevertheless, several findings - such as those comparing virulent and attenuated arenaviruses or comparing non-adapted $\mathrm{EBOV}$ with $\mathrm{MA}-\mathrm{EBOV}$ in vivo - lend strong support to the idea that insufficient or inappropriate responses by DCs and macrophages are an important feature of infection with LASV or EBOV and resulting disease.

The interferon response. The rapid course of viral haemorrhagic fevers, particularly in non-human primates, indicates that intrinsic cellular responses of initial target cells are important determinants of disease. The three types of IFN - type I (IFN $\alpha$ and IFN $\beta$ ), type II (IFN $\gamma$ ) and type III (IFN $\lambda$ ) - coordinate a powerful barrier to virus infection through the upregulation of hundreds of IFN-stimulated genes (ISGs) that directly or indirectly suppress virus replication ${ }^{42}$. In the context of both EBOV and LASV, the importance of the type I IFN response in resistance to infection is demonstrated by the fact that mice deficient for the type I IFN receptor succumb to lethal infection ${ }^{43-46}$. Further support for the importance of type I IFN comes from the comparison of cytokine

\section{Box 2 | Vaccine strategies for EBOV and LASV that induce protective immune responses}

Whereas multiple vaccine candidates for Ebola virus (EBOV) are now in human phase I-III clinical trials ${ }^{129-131}$, only a limited number of Lassa virus (LASV) vaccine approaches are under development ${ }^{130}$ (Supplementary information S1 (table)). Here, we focus on the current most promising approaches.

For EBOV, two approaches are undergoing clinical trials in West Africa. The first approach is the recombinant chimpanzee adenovirus ( $\mathrm{rChAd/EBOV)}$ in combination with modified vaccinia virus Ankara (MVA-BN-Filo), both expressing the EBOV glycoprotein (GP). Vaccination with $\mathrm{rChAd/EBOV}$ alone or followed by a booster immunization with MVA-BN-Filo protected $100 \%$ of macaques from lethal EBOV infection ${ }^{132}$. Vaccination with $\mathrm{rChAd/EBOV}$ elicited both cellular and humoral immune responses. In human clinical trials of this vaccine, both antibodies and CD4 ${ }^{+}$and CD8 ${ }^{+}$ memory $T$ cells specific for the EBOV antigens (immunogens) reached comparable levels to those observed in protected non-human primates ${ }^{133,134}$. The second approach uses vesicular stomatitis virus expressing EBOV GP (VSV-EBOV). This vaccine is protective after administration of a single dose in as few as 7 days in non-human primates ${ }^{135,136}$, and antibodies have been identified as being crucial for protection ${ }^{123}$. Furthermore, this vaccine was effective within 10 days of administration in humans and it protected individuals in Guinea from EBOV infection using a ring vaccination protoco ${ }^{26}$. The rapid protection provided by VSV-EBOV is likely due to strong stimulation of innate immune responses followed by the development of antigen-specific antibodies ${ }^{136}$.

The VSV platform has also been used to develop a LASV vaccine. A single dose of VSV-Lassa-glycoprotein precursor (GPC) protects cynomolgus macaques from clinical disease. This vaccine elicited cellular and humoral immune responses, including neutralizing antibodies ${ }^{105}$. In a follow-up study, it was shown that VSV-Lassa-GPC has the potential to protect against different genetic clades of LASV ${ }^{137}$. Another promising vaccine approach is based on a reassortant virus between Mopeia virus (MOPV) and LASV, known as ML29 (REF. 138). This vaccine consists of the nucleoprotein (NP) and GPC of LASV and the polymerase (L) and zinc-binding matrix protein (Z) of MOPV. Cell-mediated immunity in response to ML29 was identified as being crucial for protection against LASV in guinea pigs and non-human primates ${ }^{139}$. Furthermore, similarly to VSV-Lassa-GPC, ML29-vaccinated immunocompromised animals developed immune responses to a level previously associated with protection against LASV challenge ${ }^{140}$. 
responses between non-human primates that survive LASV infection and those that succumb to infection. IFNa expression was strongly upregulated within the first few days of infection in surviving animals, but was undetectable in the blood days later. By contrast, IFNa expression was only upregulated at late time points before death in non-survivors ${ }^{47}$.

ISGs that directly suppress virus replication recognize components of the virus, resulting in viral destruction or impediment of function. For example, tetherin (also known as BST2) is an ISG that is reported to prevent the budding and egress of both EBOV and LASV from the cell ${ }^{48,49}$. However, ISGs also orchestrate adaptive immune responses that are required for virus clearance. Here, initial sensing of virus infection and the production of IFNs are required for macrophages and DCs to upregulate expression of co-stimulatory molecules that are necessary for antigen presentation and for priming $\mathrm{T}$ cell responses. Following virus entry into target cells, $\mathrm{EBOV}$ is sensed by host pattern-recognition receptors, of which the retinoic acid-inducible gene-I (RIG-I)-like receptors (RLRs) - RIG-I and melanoma differentiation-associated protein 5 (MDA5) - seem to be particularly important ${ }^{50}$. Activation of mitochondrial antiviral signalling protein (MAVS; also known as VISA, IPS1 and CARDIF) downstream of RLRs results in the formation of signalling platforms to activate the key

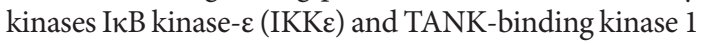
(TBK1), which in turn activate nuclear factor- $\kappa \mathrm{B}$ (NF$\kappa B$ ) and IFN regulatory factor 3 (IRF3) and/or IRF7, respectively. These transcription factors are responsible for driving the expression of IFNs and pro-inflammatory cytokines $^{51}$. Secreted IFNs then signal to cells via the Janus kinase-signal transducer and activator of transcription (JAK-STAT) pathway to amplify ISG expression and to upregulate the expression of co-stimulatory molecules by $\mathrm{DCs}^{52}$.

Viral antagonism. The importance of these innate immune pathways in viral control is supported by the multiple mechanisms that are encoded by vertebrate viruses to evade these responses ${ }^{53}$. Specifically, EBOV uses the viral protein VP35 (REF. 54) to block MAVS-dependent signalling through several mechanisms ${ }^{50}$, including: binding of viral double-stranded RNA (dsRNA) to prevent its recognition by RIG-I and MDA5 (REFS 55-57); binding of a positive regulator of RIG-I known as PACT ${ }^{58}$; functioning as a decoy substrate for IKK $\varepsilon$ and TBK1 to prevent IRF3 activation ${ }^{59}$; and promoting IRF3 and IRF7 sumoylation to impair their transcriptional activity ${ }^{60,61}$ (FIG. 2). The molecular biology of these interactions has been reviewed in detail elsewhere ${ }^{50}$. The activities of VP35 suppress the maturation of infected $\mathrm{DCs}^{62}$, thus disrupting the crucial bridge between innate and adaptive immune responses. Furthermore, EBOV VP24 functions in infected cells to antagonize signalling by secreted IFNs by binding to karyopherin- $\alpha$ (KPNA) proteins and inhibiting their association with STAT1 (REFS 63-65). This prevents the nuclear localization of STAT1 and hence ISG expression. This viral evasion strategy is likely to curtail signalling by all three types of IFN, which involves a crucial role for STAT1 in each case. As the specific IFNs have varied roles in immune responses, including direct antiviral activity, immune modulation and maintaining endothelial barrier integrity, the antagonism of KPNA by EBOV may have far-reaching implications for viral pathogenesis. The functions of VP24 and VP35 in antagonizing the innate immune response have been shown, using reverse genetics, to be important for EBOV pathogenesis in $v i v o^{66,67}$, thus demonstrating the crucial importance of viral evasion of innate immune signalling in establishing virus replication and immune dysfunction.

EBOV has other mechanisms to manipulate intrinsic cellular responses in addition to the direct suppression of viral RNA sensing and of STAT1 activity (FIG. 2). The TYRO3/AXL/MER (TAM) family of receptor tyrosine kinases have two functions in immunity: the recognition of phosphatidylserine on the outside of apoptotic cells, resulting in their phagocytosis; and the negative regulation of pathogen sensing and type I IFN signalling, most notably through the upregulation of expression of suppressor of cytokine signalling proteins (SOCS1 and SOCS3), which are potent suppressors of JAK activity ${ }^{68,69}$. T cell immunoglobulin mucin domain protein 1 (TIM1) is also a phosphatidylserine receptor ${ }^{70}$. EBOV cloaks its envelope with phosphatidylserine to engage both TAM receptors (as shown for AXL and MER) and TIM1, in a process termed apoptotic mimicry, in order to bind target cells $\mathrm{s}^{71}$; MER binding has been shown to be important for infection of macrophages ${ }^{72}$. Binding of TAM receptors on target cells also triggers intracellular signalling that results in the upregulation of SOCS proteins and thereby the suppression of signalling by type I $\mathrm{IFN}^{73}$. The upregulation of SOCS proteins through this and other mechanisms (including Toll-like receptor 4 engagement by viral glycoprotein $(\mathrm{GP})^{74}$ ) probably has additional benefits for the virus through the direct binding of SOCS3 to VP40, which promotes virus budding ${ }^{75}$. Therefore, the activation of pathways that suppress IFN signalling may affect EBOV replication directly, beyond the obvious advantage in terms of preventing ISG expression. Finally, EBOV antagonizes the action of individual ISGs, for example protein kinase R (PKR) and tetherin. Interestingly, the viral protein responsible for the suppression of PKR is VP35. As PKR binds dsRNA, it might be expected that the RNA-binding function of VP35 competes for recognition of viral RNA by PKR. However, mutant forms of VP35 that are unable to bind dsRNA retain their function in PKR antagonism ${ }^{76,77}$, which indicates that additional mechanisms exist ${ }^{50}$. As a second example, tetherin functions by binding to VP40 and retaining virus-like particles at the cell surface during budding. However, EBOV GP antagonizes the actions of tetherin to promote virus budding and cellular release. This function of GP maps to the glycan cap and requires the GP transmembrane domain ${ }^{78-80}$, although the precise mechanism of tetherin antagonism by GP is not completely understood.

Similar to EBOV, LASV also antagonizes IFN responses by preventing viral RNA sensing, which results in suppressing the maturation of infected 


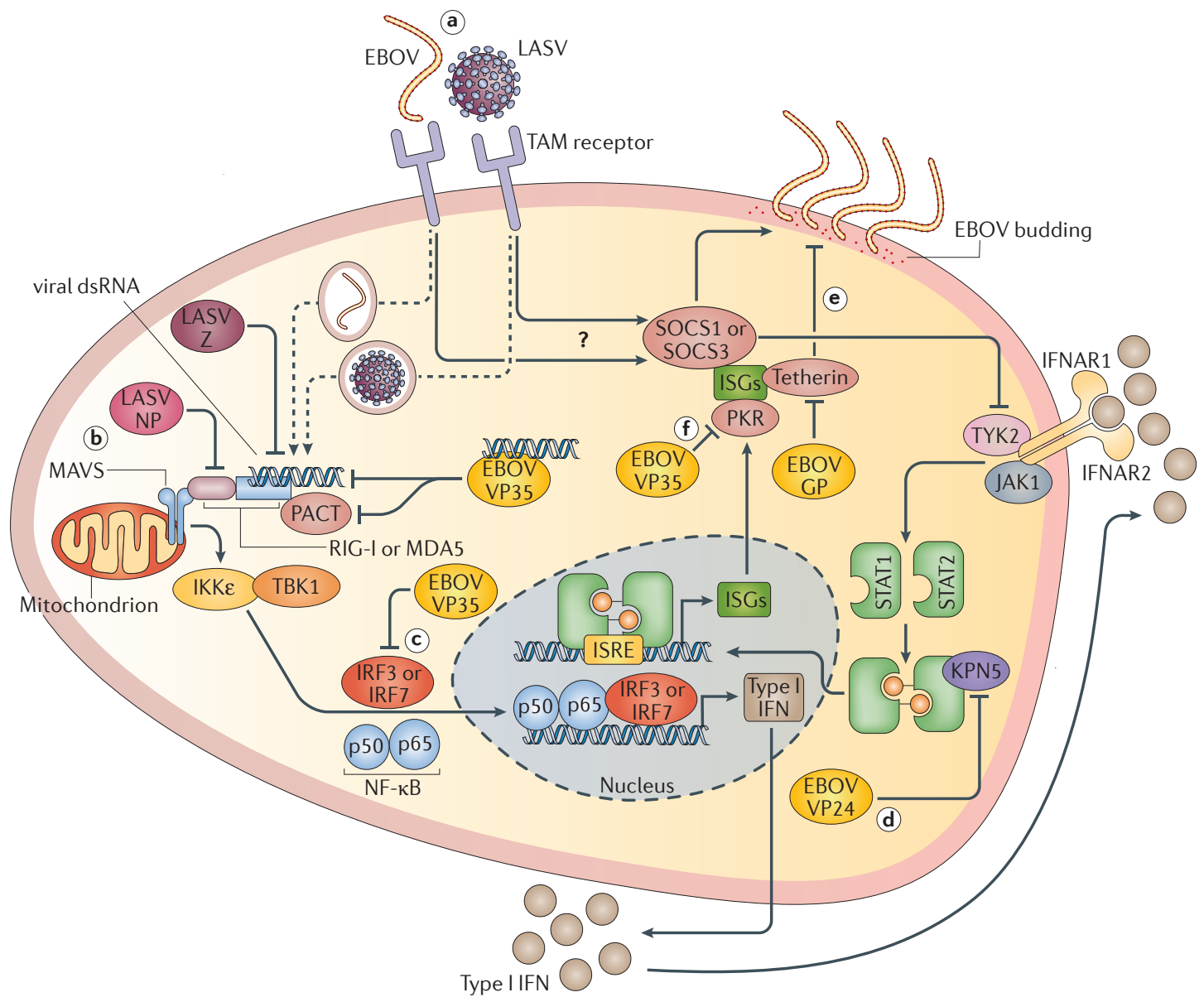

Figure 2 | Innate immune activation and antagonism by EBOV and LASV. Following virus entry and uncoating, viral double-stranded RNA (dsRNA) is recognized by the retinoic acid-inducible gene-I- mitochondrial antiviral signalling protein (RIG-I-MAVS) or melanoma differentiation-associated protein 5 (MDA5)-MAVS pathway, leading to activation of the kinases $\mid \kappa B$ kinase- $\varepsilon(\mathrm{IKK} \varepsilon$ ) and TANK-binding kinase 1 (TBK1), and of the transcription factors nuclear factor- $\kappa B$ (NF- $\kappa B$ ) and interferon (IFN) regulatory factor 3 (IRF3) and/or IRF7. Transcription factor activation drives expression of type IIFN that, following secretion from the infected cell, binds to the type I IFN receptor (IFNAR1-IFNAR2) and activates the Janus kinase-signal transducer and activator of transcription (JAK-STAT) signalling cascade. Activated STAT transcription factors bind to IFN-stimulated response elements (ISREs) upstream of target genes to amplify the expression of hundreds of IFN-stimulated genes (ISGs), including protein kinase R (PKR) and tetherin. Various strategies are used by Ebola virus (EBOV) and Lassa virus (LASV) to antagonize these innate immune signalling pathways. a | Engaging TAM (TYRO3/AXL/ MER) receptors to bind cells and promote virus entry (dashed line) also upregulates the expression of suppressor of cytokine signalling (SOCS) proteins that negatively regulate JAK-STAT signalling. SOCS proteins also promote EBOV budding. $\mathbf{b}$ | Viral proteins suppress RIG-I-like receptor (RLR) activation by preventing the recognition of viral dsRNA, which is mediated by EBOV viral protein 35 (VP35) or LASV zinc-binding matrix protein (Z), or by inhibiting RLR activity, which is mediated by EBOV VP35 and LASV nucleoprotein (NP). VP35 also binds and inhibits PACT, a positive regulator of RIG-I. c | EBOV VP35 inhibits the activation of IKKE and TBK1, and it suppresses transcriptional activity of IRF3 and IRF7. d | EBOV VP24 binds karyopherin 5 (KPN5) and prevents the nuclear localization of STAT1. e | The ISG tetherin prevents budding of both EBOV and LASV, but this activity is counteracted by the actions of EBOV glycoprotein (GP). f | The ISG PKR is counteracted by EBOV VP35. TYK2; tyrosine kinase 2.

DCs (FIG. 2). The LASV small matrix protein $Z$ inhibits signalling through both RIG-I and MDA5 by binding to the caspase-recruitment domain (CARD) of both proteins and preventing its interaction with the downstream adaptor $\mathrm{MAVS}^{81}$. In addition, LASV nucleoprotein (NP) uses at least two strategies to suppress the expression of type I IFN. First, NP encodes an exonuclease activity that has remarkable specificity for viral dsRNA, resulting in its degradation ${ }^{82,83}$. This activity presumably scavenges viral RNA that is not directly involved in replication or transcription and translation, and prevents its recognition by RLRs. Second, NP binds to IKKe and prevents its activation of the downstream transcription factors IRF3 and NF- $\kappa B^{84,85}$. Hence, both LASV and EBOV use strategies to prevent the direct recognition of the viral RNA by RLRs, in addition to mediating specific suppression of RLR-dependent signalling downstream of RNA sensing. Furthermore, LASV also uses the TAM receptors AXL and TYRO3 as auxiliary receptors to dystroglycan for viral entry ${ }^{86}$. Importantly, activation of the TAM 
receptors is required for LASV entry, which indicates that LASV may use the same apoptotic mimicry strategy as EBOV to upregulate SOCS expression and suppress signalling through the type I IFN receptor. However, these compelling in vitro findings require further investigation in vivo to determine the relevance of this pathway to the pathogenesis of LASV and EBOV.

\section{T cell-mediated immune responses}

$T$ cell apoptosis in EVD. In addition to the improper priming of T cells by infected, immature APCs, lymphocyte apoptosis is thought to be a central feature of EVD. Lymphocyte apoptosis occurs in secondary lymphoid tissues upon experimental infection of non-human primates with EBOV ${ }^{82}$. However, EBOV does not show tropism for lymphocytes ${ }^{87}$, which indicates that lymphocyte apoptosis is due to some type of bystander-cell response to infection. Similarly, analysis of peripheral blood mononuclear cells (PBMCs) in non-human primates suggests that there is a depletion of circulating $\mathrm{T}$ cells and natural killer (NK) cells ${ }^{88}$. Lymphocyte apoptosis would have obvious negative effects on the generation of an effective adaptive immune response, and this is thought to be one of the key ways in which EBOV disarms the immune system.

Lymphocyte apoptosis is also observed in humans during EVD, but the mechanisms by which this occurs are poorly understood. Analysis of the peripheral blood of three patients sampled within a day before death from EVD showed a reduced percentage of $\mathrm{CD}^{+} \mathrm{T}$ cells, compared with healthy controls, and high levels of expression of CD95 (also known as FAS) on T cells ${ }^{89}$. The absolute numbers of $\mathrm{T}$ cells were not determined, and reduced percentages could indicate the egress of $\mathrm{T}$ cells from the blood to sites of virus replication and tissue destruction. Although the observed high levels of expression of CD95 might indicate cells that are poised for apoptosis, CD95 expression is also upregulated in the early phases of $\mathrm{T}$ cell activation and functions as a marker for cell survival in some contexts ${ }^{90}$; a more important regulator of T cell apoptosis is the expression level of CD95 ligand (CD95L). Interpreted differently, therefore, these results could suggest that robust $\mathrm{T}$ cell activation occurs before patient death from EVD, as has been described more recently in patients with $\mathrm{EVD}^{91}$. Unfortunately this data set is extremely small, owing to sample availability, including control samples ${ }^{89}$. However, additional evidence of $\mathrm{T}$ cell apoptosis during EBOV infection comes from the analysis of patient PBMCs from EBOV outbreaks in Gabon ${ }^{92}$. Here, fatal cases of EVD showed indicators of $\mathrm{T}$ cell activation at early time points during EVD, followed by large decreases in the levels of CD3, CD8 and T cell receptor mRNA and downregulation of the anti-apoptotic protein BCL-2 compared with patients who would ultimately survive. In addition, DNA fragmentation of leukocytes, which is indicative of apoptosis, has been observed in the blood of patients ${ }^{93}$. Again, these data are suggestive of apoptotic events, but the extravasation of activated $\mathrm{T}$ cells to tissues in fatal cases could contribute to the decreased percentage of peripheral $\mathrm{T}$ cells and reduced mRNA levels that have been observed.
Thus, although there is strong evidence from animal models that EBOV induces extensive lymphocyte apoptosis, the limited availability of human samples, particularly from lymphoid tissues, makes it less clear whether this is a feature of human disease. Conversely, LASV infection does not lead to conspicuous immune cell apoptosis and does not induce apoptotic signalling ${ }^{35}$. Thus, if apoptosis is a major feature of EVD in humans, it probably distinguishes EVD from Lassa fever (FIG. 3).

Early T cell activation correlates with EVD. Adaptive immune responses are poorly described in humans infected with EBOV owing to a scarcity of tissue samples. Histological preparations of tissues from infected individuals are almost non-existent and what is known comes from the analysis of cytokine expression in sera and from a small number of PBMC samples of infected individuals from a limited number of outbreaks ${ }^{87,89,90}$. Pathogenesis studies of EBOV have been carried out in macaques that describe the kinetics of immune cell responses ${ }^{88}$. However, the relatively short time to death in this model makes it difficult to study the adaptive immune response and to correlate observations with differential outcomes. This is not the case for LASV, which is not uniformly lethal in all non-human primate models.

In humans, serum levels of IFN $\gamma$ and other cytokines, such as IL- 2 and IL-4, are measured as indicators of the quantity and quality of lymphocyte responses. IFN $\gamma$ is preferentially produced by T cells and NK cells and is a marker for T helper $1\left(\mathrm{~T}_{\mathrm{H}} 1\right)$ cell-skewed responses ${ }^{94}$. In a study of EBOV-infected patients from Gabon, serum levels of IFN $\gamma$ and IL-2 were increased in fatal cases compared with survivors, which indicates higher levels of $\mathrm{T}$ cell activation in the former ${ }^{95}$. However, the same patients had increased levels of IL-10, particularly late in infection. IL-10 is strongly anti-inflammatory and immunosuppressive, and it may be produced as a counter to early inflammation defined by IFN $\gamma$ and IL-2. Furthermore, high levels of IFN $\gamma$ can contribute to $\mathrm{T}$ cell apoptosis. Although this study did not measure lymphocyte populations in the blood, increased levels of IFN $\gamma$ could lead to decreased T cell responses via apoptosis and thereby contribute to ineffective immune responses ${ }^{94}$. These findings were supported by a second study showing that survivors of EBOV infection from Gabon had low levels of mRNAs encoding IFN $\gamma$ and the T cell activation markers CD28, CD95 and CD95L during the symptomatic phase that increased as the patients convalesced $^{92}$. By contrast, fatal cases of EBOV infection were associated with higher levels of these mRNAs early in the symptomatic phase, followed by a decrease. These findings indicate that fatal cases might have had an early $\mathrm{T}$ cell response that subsided, or more likely was counteracted by apoptotic or other immunosuppressive events, in the days before death. Both studies ${ }^{92,95}$ found that high levels of IFN $\gamma$ early in disease correlated with a fatal outcome. Analysis of the same patients revealed that increased levels of IL-1 receptor antagonist (IL-1RA) and, as in the previous study, of IL-10 corresponded with a fatal outcome ${ }^{93}$. In patients infected with Sudan 

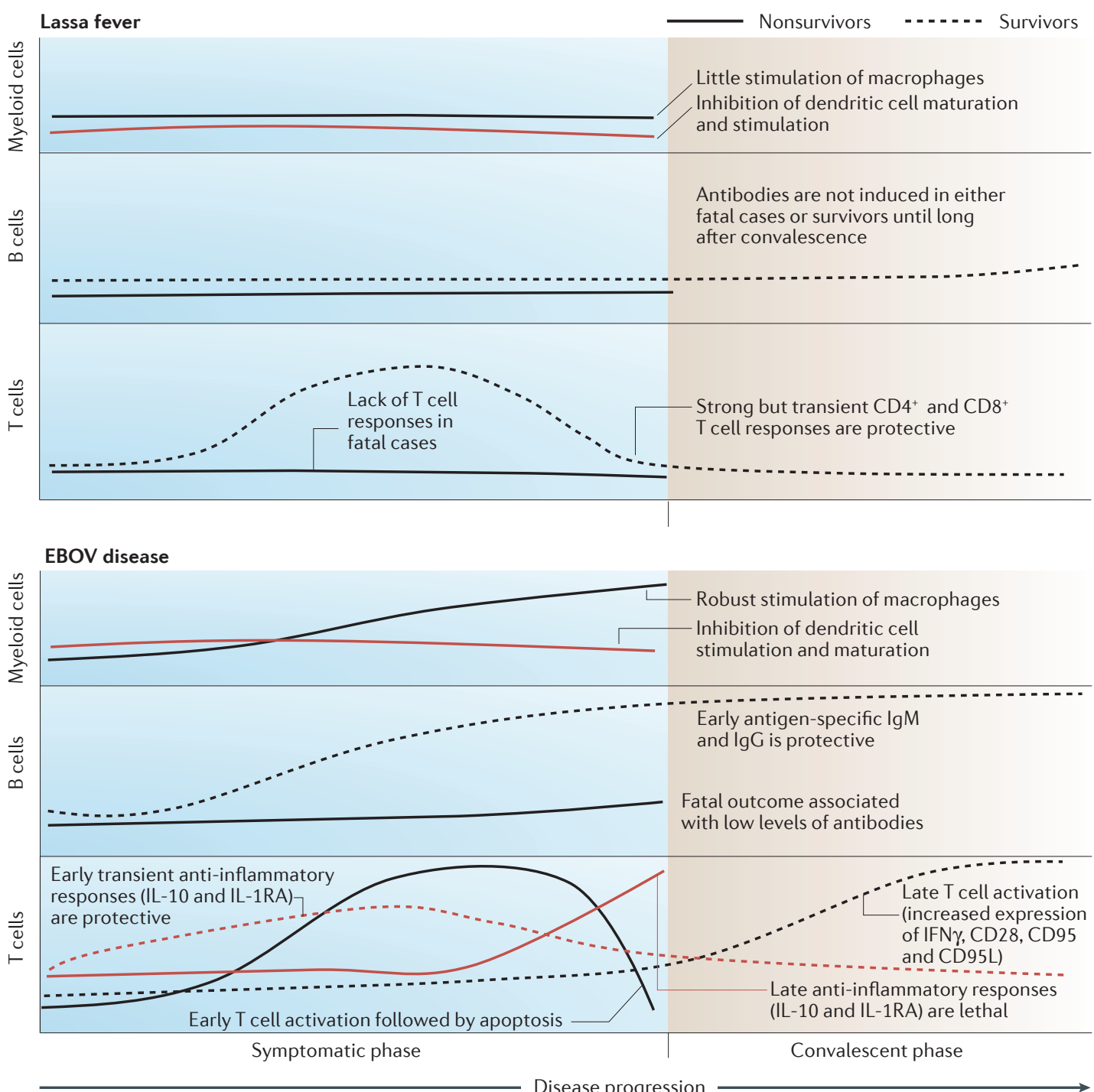

Figure 3 | Disparate immunological events that correlate with fatality or survival from infection with LASV or EBOV. Myeloid cells (dendritic cells (DCs) and monocytes and macrophages) are the initial target cells of both Ebola virus (EBOV) and Lassa virus (LASV), and both viruses inhibit DC maturation and DC responses; however, LASV inhibits macrophage responses, whereas EBOV activates these cells. This suggests that dysregulation of DC responses may be central to disease. B cell responses, as measured by antibody production, show that early production of lgG and lgM is protective for EBOV infection, yet dispensable for protection against LASV. This implicates $C D 8^{+} T$ cell responses as being important for survival from LASV infection. Indeed, T cell responses are important for surviving both EBOV and LASV. In the context of EBOV, survival correlates with a balanced immune response - with initial weak inflammatory responses combined with measurable anti-inflammatory responses in the early symptomatic phase - and with the development of a robust $T$ cell response. Conversely, an ineffective response is characterized by an early hyperactive T cell response that progresses to an anti-inflammatory response, defined by interleukin-10 (IL-10) upregulation and lymphocyte apoptosis in the symptomatic phase. By contrast, protective responses to LASV infection consist of a transient yet strong inflammatory response, whereas severe disease is associated with very weak T cell responses. CD95L, CD95 ligand; IFN, interferon; IL-1RA, IL-1 receptor antagonist.

ebolavirus, IFNa levels were higher in the serum of survivors compared with fatal cases, which might indicate that a beneficial innate immune response was initiated in survivors; similarly to infection with EBOV, levels of IL-6, CXCL8, IL-10 and CCL2 were higher in fatal cases of Sudan ebolavirus infection ${ }^{89,96}$. In these samples, IFN $\gamma$ levels were unchanged in both survivors and nonsurvivors, perhaps owing to differences between EBOV and Sudan ebolavirus or to host genetics.
These results support a model whereby early T cell activation (defined by IFN $\gamma$ and IL- 2 production) followed by downregulation of $\mathrm{T}$ cell responses by cytokines such as IL-10 and IL-1RA and/or by apoptosis late in the disease results in a lethal outcome of EBOV infection (FIG. 3). Survivors have lower levels of $\mathrm{T}$ cell activation early after infection that transition to an inflammatory response without the corresponding anti-inflammatory response. A key to pathogenesis 
might be production of IL-10, which is one of the most potent anti-inflammatory molecules and can regulate immune responses by inhibiting $\mathrm{T}_{\mathrm{H}} 1$ cells and NK cells, by directly inhibiting the production of IFN $\gamma$, and by stimulating the activity of anti-inflammatory regulatory T cells ${ }^{97,98}$. Myeloid cells, including macrophages, $\mathrm{mDCs}$ and monocytes, are primary producers of IL-10 following pattern-recognition receptor engagement ${ }^{99}$. The source(s) of IL-10 during EBOV and Sudan ebolavirus infection is not known, but its importance in EBOV infection has been investigated in mice, whereby abolishing IL-10 reduces mortality rates ${ }^{100}$. In asymptomatic EBOV-infected humans, IL-10 expression is upregulated early and transiently, whereas in fatal cases, IL-10 upregulation occurs later after infection ${ }^{101}$. Similar to IL-10, IL-1RA has anti-inflammatory effects by blocking the normal pro-inflammatory functions of IL- $1 \alpha$ and IL-1 $\beta$; the upregulation of IL-1RA late in disease could be detrimental by inhibiting antiviral $\mathrm{T}$ cell responses ${ }^{102}$.

A more recent examination of four patients who survived EBOV infection after contracting EVD during the 2013-2016 West African outbreak showed that these patients had strong induction of cellular and humoral immune responses, which brings into question the dogma that EBOV infection results in a generalized immunosuppression and in lymphocyte apoptosis ${ }^{91}$. This study showed substantial increases in the number of $\mathrm{T}$ cells and their expression of activation markers. However, a major difference between this study and previous outbreak studies is that these patients were transported to a clinical centre in the US and received substantial supportive care as well as, potentially, treatments such as monoclonal antibody therapy. This care and treatment probably facilitated their recovery and enabled sampling to be continued for far longer than was previously possible. A longitudinal study of 204 patients from the recent West African outbreak showed that expression of the inhibitory molecules cytotoxic T lymphocyte antigen 4 (CTLA4) and programmed cell death 1 (PD1) on both $\mathrm{CD}^{+}$and $\mathrm{CD}^{+} \mathrm{T}$ cells was significantly and continuously upregulated in fatal cases but only transiently upregulated in survivors ${ }^{103}$. This occurred independently of $\mathrm{T}$ cell activation, which was similar for both groups. This study also found that levels of IL-10 were higher in the fatal cases of EVD (FIG. 3).

Early $T$ cell responses correlate with survival in Lassa fever. Lassa fever is characterized by the depletion of $\mathrm{T}$ cells in secondary lymphoid tissues, a transient lymphopenia and decreased $\mathrm{T}$ cell proliferation, at least in macaques ${ }^{47}$. $\mathrm{T}$ cell responses, but not $\mathrm{B}$ cell responses, seem to be central for immunity to LASV (FIG. 3). This is supported by two major lines of evidence. First, T cells (both $\mathrm{CD} 4^{+}$and $\mathrm{CD} 8^{+}$) are activated early during infection, and can be detected in patients after recovery from Lassa fever, despite little or no detectable antibody responses during clinical disease and a slow increase in antibody titres long after convalescence ${ }^{104}$. Second, less direct evidence comes from the fact that vaccines that elicit $\mathrm{T}$ cell responses, but not strong antibody responses, against LASV glycoproteins are protective in non-human primates and guinea pigs, which indicates that $\mathrm{T}$ cell responses are a major determinant for protection against disease. For example, a recombinant vaccinia virus that induces strong $\mathrm{T}$ cell responses against both GP1 and GP2 of LASV, but weak antibody responses, is protective in non-human primates ${ }^{38}$. By contrast, vaccinia virus-expressed LASV NP elicited strong antibody responses but was not protective. This does not exclude the possibility that a vaccine that can elicit strong antibody responses to GP1 and GP2 would be protective, which was the case for the VSV-LassaGPC (VSV-Lassa-glycoprotein precursor) vaccine ${ }^{105}$. However, a dominant role for $\mathrm{T}$ cells in protection against LASV is not surprising given that the extensively characterized immune response to the arenavirus lymphocytic choriomeningitis virus (LCMV) in mice is largely $\mathrm{T}$ cell driven ${ }^{106}$.

In humans, strong $\mathrm{CD} 4^{+} \mathrm{T}$ cell memory responses against LASV NP can be recalled in PBMCs for up to six years after the initial infection, and $\mathrm{T}$ cell memory responses to GP are similarly long-lived ${ }^{107,108}$. In macaques, LASV infection that results in a fatal outcome is associated with a lack of demonstrable T cell activation throughout the course of disease as defined by expression of the T cell activation markers CD69 and CD25 and the proliferation marker Ki67. By contrast, animals that survived LASV infection had transient but strong activation and proliferation of $\mathrm{CD} 4^{+}$and $\mathrm{CD} 8^{+}$ $\mathrm{T}$ cells between 5 and 15 days after inoculation ${ }^{47}$. An in vitro comparison between LASV and MOPV showed that $\mathrm{T}$ cell responses were much more robust when autologous naive human $\mathrm{T}$ cells were co-cultured with DCs pulsed with MOPV compared with DCs pulsed with LASV ${ }^{109}$. This clearly demonstrates a biological difference between the pathogenic and non-pathogenic viruses in terms of their ability to be efficiently presented by APCs and to activate T cells. Furthermore, these results are unlikely to be skewed by differences in epitope-MHC restriction between MOPV and LASV because previous infection of non-human primates with MOPV confers protection upon challenge with LASV ${ }^{110}$.

\section{Humoral immunity}

Antibody responses to EBOV correlate with protection. For EBOV, early studies of passive immunotherapy by antibody treatment showed largely disappointing results ${ }^{111-113}$, which indicated that cellular immunity might have a more important role than humoral immunity in protection. However, more recently, several studies have shown that antibodies specific for EBOV GP can afford protection, in the form of either vaccineinduced antibody responses or immunotherapy, and that there is a strong correlation between survival after infection with EBOV and early and strong antibody production in humans ${ }^{92}$.

Antibodies were used as a treatment strategy for EVD in humans as early as 1995, when eight people received blood transfusions from convalescent patients ${ }^{114}$. In this case, seven patients ultimately survived the disease, although it is difficult to determine whether antibody 
administration had an effect as these patients may have already been in a recovery phase before antibody transfer, and additional constituents in donated blood may also have had beneficial effects. Recent studies observed a risk of death of $31 \%$ for patients treated with convalescent plasma compared with $38 \%$ for control patients, again suggesting limited efficacy of antibody therapy ${ }^{115}$. Early studies using monoclonal antibodies in animal models had mixed success. Although a single monoclonal antibody directed against EBOV GP (KZ52) could protect guinea pigs from death, it was ineffective at inhibiting viral replication and preventing disease in macaques ${ }^{116,117}$. Recently, a cocktail of three monoclonal antibodies (MB-003) that recognize GP proved efficacious when administered postchallenge in macaques ${ }^{118,119}$. Another cocktail of three antibodies against GP (ZMapp), sharing components of MB-003, reversed disease when given at three-day intervals starting five days after EBOV inoculation in macaques ${ }^{24}$. These antibodies have been subsequently used to treat humans during the West African outbreak $^{120}$. More strikingly, a single monoclonal antibody for GP isolated from a survivor of the $1995 \mathrm{EVD}$ outbreak in Kikwit, DRC, was effective at preventing disease in macaques when administered five days postinoculation ${ }^{121}$. These data are promising as the control animals had to be euthanized between days six and nine in this study. This antibody is neutralizing as well as able to mediate antibody-dependent cell-mediated cytotoxicity (ADCC) in vitro, which possibly accounts for its potency. However, this antibody did not prevent virus replication despite preventing disease, which suggests the possibility that virus escape mutants could arise. Further studies regarding monoclonal antibody therapies are needed to address both the mechanisms underlying protection from infection and the potential for virus escape mutants to arise and, if so, the effects of any mutations on virus fitness.

Comparing antibody responses to EBOV in humans with disparate outcomes revealed that survivors have a much increased antibody response compared with fatal cases of EVD, and people who experienced asymptomatic infection have high antibody levels ${ }^{92,101,122}$. In addition, protection provided by the VSV-based vaccine, which is highly efficacious in non-human primates and shows promise in humans, is mediated by $\mathrm{CD} 4^{+}$ $\mathrm{T}$ cell-dependent antibody responses, which shows that antibody responses can be protective ${ }^{123}$. Although these data strongly suggest that antibodies are important for a favourable outcome in EBOV infection, $\mathrm{T}$ cell responses are required for efficient antibody class switching and affinity maturation of IgG. Thus, $\mathrm{CD} 4^{+}$ $\mathrm{T}$ cells are likely equally important in mounting a strong humoral response.

Antibody-dependent cell-mediated cytotoxicity (ADCC). A cell-mediated immune response whereby cells bound by specific antibodies are lysed, primarily by natural killer cells or neutrophils. phases of recovery and antibody titres often increase only late during convalescence. These observations support the vaccine studies mentioned above, which concluded that antibodies are dispensable for protection against LASV because they are not induced by some vaccines that are still efficacious ${ }^{35}$. Furthermore, the dominance of cellular immunity in protection is supported experimentally in macaques infected with LASV ${ }^{47}$. However, non-neutralizing antibodies could aid in the clearance of LASV by one of several functions, such as ADCC, complement fixation and opsonisation-mediated phagocytosis. Thus, T cell responses are certainly important for immunity to LASV, but antibody responses could provide additional immune support, particularly for protection against re-infection.

\section{Conclusions and future directions}

In summary, EBOV acts to shut down DC responses while at the same time hyper-activating macrophages early in infection. This effect is associated with a strong inflammatory response to early EBOV infection that transitions to an anti-inflammatory response with widespread T cell apoptosis later in infection. Death of the host probably results from the high levels of lytic virus replication in addition to the inflammatory response driving coagulation abnormalities. Survivors of EBOV infection are better able to regulate the antiinflammatory response than non-survivors and they produce protective antibodies. In the case of LASV infection, both DC and macrophage responses are antagonized, and $\mathrm{T}$ cell responses are very weak in fatal cases of Lassa fever, whereas survivors mount a strong but transient $\mathrm{T}$ cell response. In contrast to EVD, B cell responses seem not to be important for natural immunity leading to survival from LASV infection, although neutralizing antibodies are made by survivors of infection, and these might have therapeutic applications ${ }^{125}$. Thus, although both EVD and Lassa fever are defined as haemorrhagic fevers, clear differences exist in both the immune responses that are elicited and in the responses that mediate a favourable outcome.

A central question in understanding the pathogenesis of viral haemorrhagic fever is what are the key mechanisms that drive disease? Inflammatory responses certainly contribute to the endpoint of disease, with the hallmark of EVD being a 'cytokine storm' that is thought to promote coagulation abnormalities and vascular leakage, but it is not clear whether this inflammation is an early driver of pathology. Alternatively, is immune suppression directly resulting from virus infection of key cells or indirectly from the expression of anti-inflammatory cytokines the early driver of disease? These seemingly divergent phenomena are probably regulated in both a spatial manner (according to cell and tissue type) and a temporal manner. Therefore, careful studies designed to understand how early events in infected myeloid cells initiate inflammatory or protective responses are warranted. These types of studies will probably reveal new functions of individual viral proteins in triggering or suppressing cellular signalling pathways to alter immune responses. 
The ideal immunotherapeutic would be one that provides benefit in cases of haemorrhagic fever regardless of the infectious agent. However, this may be very difficult to achieve and may even be detrimental given the clear differences in immune activation versus immune suppression observed in EVD and Lassa fever, respectively. Future research to fill the gaps in our knowledge may determine how therapeutic targeting of RLR activation, production of IFNs and pro- and anti-inflammatory cytokines could affect the outcome of disease. It may be that therapeutic targets are not compatible between these two diseases and that efforts should be focused on boosting the natural immune responses that are known to be protective, such as antibody responses in EVD and cytotoxic T lymphocyte (CTL) responses in Lassa fever. A complementary approach may be to augment the responses that are not strongly induced early in natural infection, including CTL responses in EVD and the humoral response to LASV. Furthermore, information regarding the mechanisms of control of virus replication and inflammation may be garnered from comparative studies in animal models that do not result in disease. Examples here include the comparison of non-adapted EBOV with MA-EBOV, comparison of virulent and attenuated virus strains, or examination of virus replication in reservoir species of bats (the likely reservoir of EBOV) and rodents (M. natalensis, the reservoir of LASV); for example, related studies have yielded insight into immunological control in deer mice infected with Sin Nombre virus, a hantavirus ${ }^{126}$. Finally, in order to impact public health, the development of countermeasures for EVD and Lassa fever needs to be intensified, guided by results from basic research. Existing approaches such as antivirals with promising preclinical data (for example, small molecule inhibitors and antibodies) need to be moved into human trials and towards licensure. However, additional new approaches (such as next-generation vaccines and additional small-molecule inhibitors) need to be developed and evaluated both at the preclinical level and in phase I human trials. The failure to advance these countermeasures to early clinical trials in humans now will be a major limiting factor in the implementation of any promising approach and will ensure that the next large outbreak of viral haemorrhagic fever will catch us off-guard again.
1. Paessler, S. \& Walker, D. H. Pathogenesis of the viral hemorrhagic fevers. Annu. Rev. Pathol. 8, 411-440 (2013).

2. Baize, S. et al. Emergence of Zaire Ebola virus disease in Guinea. N. Engl. J. Med. 371, 1418-1425 (2014).

3. Feldmann, F., Sanchez, A. \& Geisbert, T. W. in Fields Virology 6th edn (eds M Knipe, D. \& Howley, P. M.) 923-956 (Lippincott Williams and Wilkins, 2013).

4. Kuhn, J. H. et al. in Virus Taxonomy: Ninth Report of the International Committee on Taxonomy of Viruses (eds King, A. M. Q., Adams, M. J., Carstens, E. B., \& Lefkowitz, E. J.) 665-671 (Elsevier/Academic Press, 2011).

5. Frame, J. D., Baldwin, J. M. Jr, Gocke, D. J. ¿ Troup, J. M. Lassa fever, a new virus disease of man from West Africa. I. Clinical description and pathological findings. Am. J. Trop. Med. Hyg. 19, 670-676 (1970).

6. Fichet-Calvet, E. \& Rogers, D. J. Risk maps of Lassa fever in West Africa. PLoS Negl. Trop. Dis. 3, e388 (2009).

7. Sogoba, N., Feldmann, H. \& Safronetz, D. Lassa fever in West Africa: evidence for an expanded region of endemicity. Zoonoses Public Health 59 (Suppl. 2), 43-47 (2012).

8. Ogbu, O., Ajuluchukwu, E. \& Uneke, C. J. Lassa fever in West African sub-region: an overview. J. Vector Borne Dis. 44, 1-11 (2007).

9. Paweska, J. T. et al. Nosocomial outbreak of novel arenavirus infection, southern Africa. Emerg. Infect. Dis. 15, 1598-1602 (2009).

10. Fisher-Hoch, S. P. et al. Review of cases of nosocomial Lassa fever in Nigeria: the high price of poor medical practice. BMJ 311, 857-859 (1995).

11. Geisbert, T. W., Strong, J. E. \& Feldmann, H. Considerations in the use of nonhuman primate models of Ebola virus and Marburg virus infection J. Infect. Dis. 212 (Suppl. 2), S91-S97 (2015).

12. Smith, D. R., Holbrook, M. R. \& Gowen, B. B. Animal models of viral hemorrhagic fever. Antiviral Res. 112, 59-79 (2014)

13. Cheng, Y. et al. Principles of regulatory information conservation between mouse and human. Nature 515 371-375 (2014).

14. Chertow, D. S. et al. Ebola virus disease in West Africa - clinical manifestations and management. N. Engl. J. Med. 371, 2054-2057 (2014). This study provides a comprehensive view of the outcome and management of patients with EVD from the 2013-2016 outbreak in Liberia.

15. Schieffelin, J. S. et al. Clinical illness and outcomes in patients with Ebola in Sierra Leone. N. Engl. J. Med. 371, 2092-2100 (2014).

16. Mattia, J. G. et al. Early clinical sequelae of Ebola virus disease in Sierra Leone: a cross-sectional study. Lancet Infect. Dis. 16, 331-338 (2016).
17. Tiffany, A. et al. Ebola virus disease complications as experienced by survivors in Sierra Leone. Clin. Infect. Dis. 62, 1360-1366 (2016).

18. Caluwaerts, S. et al. Dilemmas in managing pregnant women with Ebola: 2 case reports. Clin. Infect. Dis. 62 903-905 (2016).

19. Mupapa, K. et al. Ebola hemorrhagic fever and pregnancy. J. Infect. Dis. 179 (Suppl. 1), S11-S12 (1999).

20. Bausch, D. G. et al. Lassa fever in Guinea: I. Epidemiology of human disease and clinical observations. Vector Borne Zoonotic Dis. 1, 269-281 (2001).

21. Frame, J. D. Clinical features of Lassa fever in Liberia Rev. Infect. Dis. 11 (Suppl. 4), S783-S789 (1989).

22. McCormick, J. B. \& Fisher-Hoch, S. P. Lassa fever. Curr. Top. Microbiol. Immunol. 262, 75-109 (2002)

23. Gunther, S. \& Lenz, O. Lassa virus. Crit. Rev. Clin. Lab. Sci. 41, 339-390 (2004).

24. Qiu, X. et al. Reversion of advanced Ebola virus disease in nonhuman primates with ZMapp. Nature 514, 47-53 (2014).

This study showed that monoclonal antibody therapy can prevent the death of macaques infected with EBOV as late as $\mathbf{5}$ days after inoculation.

25. Sissoko, D. et al. Experimental treatment with favipiravir for Ebola virus disease (the JIKI Trial): a historically controlled, single-arm proof-of-concept trial in Guinea. PLoS Med. 13, e1001967 (2016).

26. Henao-Restrepo, A. M. et al. Efficacy and effectiveness of an rVSV-vectored vaccine expressing Ebola surface glycoprotein: interim results from the Guinea ring vaccination cluster-randomised trial. Lancet 386 , 857-866 (2015).

This study provides evidence of the positive effect of a ring vaccination approach using a VSV-based EBOV vaccine.

27. Geisbert, T. W. et al. Pathogenesis of Ebola hemorrhagic fever in cynomolgus macaques: evidence that dendritic cells are early and sustained targets of infection. Am. J. Pathol. 163, 2347-2370 (2003). This paper describes the cellular tropism of EBOV in vitro.

28. Ryabchikova, E. I., Kolesnikova, L. V. \& Luchko, S. V. An analysis of features of pathogenesis in two animal models of Ebola virus infection. J. Infect. Dis. 179 (Suppl. 1), S199-S202 (1999).

29. Walker, D. H., Wulff, H., Lange, J. V. \& Murphy, F. A Comparative pathology of Lassa virus infection in monkeys, guinea-pigs, and Mastomys natalensis. Bull. World Health Organ. 52, 523-534 (1975).

30. Banadyga, L., Dolan, M. A. \& Ebihara, H. Rodentadapted filoviruses and the molecular basis of pathogenesis. J. Mol. Biol. 428, 3449-3466 (2016).
31. Bray, M., Davis, K., Geisbert, T., Schmaljohn, C. $\&$ Huggins, J. A mouse model for evaluation of prophylaxis and therapy of Ebola hemorrhagic fever. J. Infect. Dis. 178, 651-661 (1998).

This paper describes the adaptation of EBOV to cause disease in mice, thereby enabling studies of EBOV in a small animal model.

32. Ebihara, H. et al. Molecular determinants of Ebola virus virulence in mice. PLoS Pathog. 2, e73 (2006).

33. Ebihara, H. et al. A Syrian golden hamster model recapitulating Ebola hemorrhagic fever. J. Infect. Dis. 207, 306-318 (2013).

34. Gibb, T. R. et al. Pathogenesis of experimental Ebola Zaire virus infection in BALB/c mice. J. Comp. Pathol. 125, 233-242 (2001).

35. Baize, S. et al. Lassa virus infection of human dendritic cells and macrophages is productive but fails to activate cells. J. Immunol. 172, 2861-2869 (2004).

36. Baize, S. et al. Role of interferons in the control of Lassa virus replication in human dendritic cells and macrophages. Microbes Infect. 8, 1194-1202 (2006).

37. Bowen, M. D., Peters, C. J. \& Nichol, S. T. Phylogenetic analysis of the Arenaviridae: patterns of virus evolution and evidence for cospeciation between arenaviruses and their rodent hosts. Mol. Phylogenet. Evol. 8, 301-316 (1997).

38. Fisher-Hoch, S. P., Hutwagner, L., Brown, B. \& McCormick, J. B. Effective vaccine for lassa fever. J. Virol. 74, 6777-6783 (2000).

39. Hawiger, D. et al. Dendritic cells induce peripheral $\mathrm{T}$ cell unresponsiveness under steady state conditions in vivo. J. Exp. Med. 194, 769-779 (2001).

40. Mahanty, S et al Cutting edge: impairment of dendritic cells and adaptive immunity by Ebola and Lassa viruses. J. Immunol. 170, 2797-2801 (2003).

41. Stroher, U. et al. Infection and activation of monocytes by Marburg and Ebola viruses. J. Virol. 75 11025-11033 (2001)

42. Schneider, W. M. Chevillotte, M. D. \& Rice, C. M. Interferon-stimulated genes: a complex web of host defenses. Annu. Rev. Immunol. 32, 513-545 (2014).

43. Brannan, J. M. et al. Interferon alpha/beta receptordeficient mice as a model for Ebola virus disease. J. Infect. Dis. 212 (Suppl. 2), S282-S294 (2015).

44. Bray, $M$. The role of the type I interferon response in the resistance of mice to filovirus infection. J. Gen. Virol. 82, 1365-1373 (2001)

45. Oestereich, L. et al. Chimeric mice with competent hematopoietic immunity reproduce key features of severe Lassa fever. PLoS Pathog. 12, e1005656 (2016).

46. Yun, N. E. et al. Functional interferon system is required for clearance of lassa virus. J. Virol. 86, 3389-3392 (2012). 
47. Baize, S. et al. Early and strong immune responses are associated with control of viral replication and recovery in lassa virus-infected cynomolgus monkeys. J. Virol. 83, 5890-5903 (2009).

This manuscript highlights the features of the cellular immune response that are protective during Lassa fever, and indicates that antibody responses are less important than cell-mediated immune responses for protection.

48. Jouvenet, $\mathrm{N}$ et al Broad-spectrum inhibition of retroviral and filoviral particle release by tetherin J. Virol. 83, 1837-1844 (2009).

49. Sakuma, T., Noda, T., Urata, S., Kawaoka, Y. \& Yasuda, J. Inhibition of Lassa and Marburg virus production by tetherin. J. Virol. 83, 2382-2385 (2009).

50. Messaoudi, I., Amarasinghe, G. K. \& Basler, C. F. Filovirus pathogenesis and immune evasion: insights from Ebola virus and Marburg virus. Nat. Rev. Microbiol. 13, 663-676 (2015)

51. Chan, Y. K. \& Gack, M. U. RIG-I-like receptor regulation in virus infection and immunity. Curr. Opin Virol. 12, 7-14 (2015).

52. MacMicking, J. D. Interferon-inducible effector mechanisms in cell-autonomous immunity. Nat. Rev. Immunol. 12, 367-382 (2012)

53. Versteeg, G. A. \& Garcia-Sastre, A. Viral tricks to grid-lock the type I interferon system. Curr. Opin. Microbiol. 13, 508-516 (2010)

54. Basler, C. F. et al. The Ebola virus VP35 protein functions as a type I IFN antagonist. Proc. Natl Acad. Sci. USA 97, 12289-12294 (2000). This is the first paper to identify EBOV VP35 as an interferon antagonist.

55. Cardenas, W. B. et al. Ebola virus VP35 protein binds double-stranded RNA and inhibits alpha/beta interferon production induced by RIG-I signaling. J. Virol. 80, 5168-5178 (2006)

56. Hartman, A. L., Towner, J. S. \& Nichol, S. T. A. C-Terminal basic amino acid motif of Zaire ebolavirus VP35 is essential for type I interferon antagonism and displays high identity with the RNA-binding domain of another interferon antagonist, the NS 1 protein of influenza A virus. Virology 328, 177-184 (2004).

57. Leung, D. W. et al. Structural basis for dsRNA recognition and interferon antagonism by Ebola VP35. Nat. Struct. Mol. Biol. 17, 165-172 (2010).

58. Luthra, P et al. Mutual antagonism between the Ebola virus VP35 protein and the RIG-I activator PAC determines infection outcome. Cell Host Microbe 14, 74-84 (2013)

59. Prins, K. C. Cardenas, W. B. \& Basler, C. F. Ebola virus protein VP35 impairs the function of interferon regulatory factor-activating kinases IKK $\varepsilon$ and TBK-1. J. Virol. 83, 3069-3077 (2009)

60. Chang, T. H. et al. Ebola Zaire virus blocks type I interferon production by exploiting the host SUMO modification machinery. PLoS Pathog. 5, e1000493 (2009).

61. Kubota, T. et al. Virus infection triggers SUMOylation of IRF3 and IRF7, leading to the negative regulation of type I interferon gene expression. J. Biol. Chem. 283 25660-25670 (2008).

62. Yen, B. C. \& Basler, C. F. Effects of filovirus interferon antagonists on responses of human monocyte-derived dendritic cells to RNA virus infection. J. Virol. 90 5108-5118 (2016)

63. Reid, S. P. et al. Ebola virus VP24 binds karyopherin alpha1 and blocks STAT1 nuclear accumulation. J. Virol. 80, 5156-5167 (2006) This is the first paper to identify EBOV VP24 as an interferon antagonist.

64. Reid, S. P., Valmas, C., Martinez, O., Sanchez, F. M. \& Basler, C. F. Ebola virus VP24 proteins inhibit the interaction of NPI-1 subfamily karyopherin alpha proteins with activated STAT 1. J. Virol. 81 13469-13477 (2007)

65. Xu, W. et al. Ebola virus VP24 targets a unique NLS binding site on karyopherin alpha 5 to selectively compete with nuclear import of phosphorylated STAT1. Cell Host Microbe 16 187-200 (2014).

66. Hartman, A. L. et al. Inhibition of IRF-3 activation by VP35 is critical for the high level of virulence of Ebola virus. J. Virol. 82, 2699-2704 (2008).

67. Prins, K. C. et al. Mutations abrogating VP35 interaction with double-stranded RNA rende Ebola virus avirulent in guinea pigs. J. Virol. 84 3004-3015 (2010).
68. Carrera Silva, E. A. et al. T cell-derived protein S engages TAM receptor signaling in dendritic cells to control the magnitude of the immune response. Immunity 39, 160-170 (2013)

69. Lemke, G. Biology of the TAM receptors. Cold Spring Harb. Perspect. Biol. 5, a009076 (2013).

70. Freeman, G. J., Casasnovas, J. M., Umetsu, D. T. \& DeKruyff, R. H. TIM genes: a family of cell surface phosphatidylserine receptors that regulate innate and adaptive immunity. Immunol. Rev. 235, 172-189 (2010)

71. Richard, A. S. et al. Virion-associated phosphatidylethanolamine promotes TIM1-mediated infection by Ebola, dengue, and West Nile viruses. Proc. Natl Acad. Sci. USA 112, 14682-14687 (2015).

72. Dahlmann, F. et al. Analysis of Ebola virus entry into macrophages. J. Infect. Dis. 212 (Suppl. 2), S247-S257 (2015)

73. Bhattacharyya, S. et al. Enveloped viruses disable innate immune responses in dendritic cells by direct activation of TAM receptors. Cell Host Microbe 14 136-147 (2013).

This paper describes the mechanism by which viruses use phosphatidylserine in the viral envelope to disable intrinsic cellular responses to infection.

74. Okumura, A., Pitha, P. M., Yoshimura, A. \& Harty, R. N. Interaction between Ebola virus glycoprotein and host toll-like receptor 4 leads to induction of proinflammatory cytokines and SOCS 1 . J. Virol. 84, 27-33 (2010)

75. Okumura, A. et al. Suppressor of cytokine signaling 3 is an inducible host factor that regulates virus egress during Ebola virus infection. J. Virol. 89, 10399-10406 (2015)

76. Feng, Z., Cerveny, M., Yan, Z. \& He, B. The VP35 protein of Ebola virus inhibits the antiviral effect mediated by double-stranded RNA-dependent protein kinase PKR. J. Virol. 81, 182-192 (2007).

77. Schumann, M., Gantke, T. \& Muhlberger, E. Ebola virus VP35 antagonizes PKR activity through its C-terminal interferon inhibitory domain. J. Virol. 83 8993-8997 (2009).

78. Gustin, J. K., Bai, Y., Moses, A. V. \& Douglas, J. L. Ebola virus glycoprotein promotes enhanced viral egress by preventing Ebola VP40 from associating with the host restriction factor BST2/tetherin. J. Infect. Dis. 212 (Suppl. 2), S181-S190 (2015).

79. Kaletsky, R. L., Francica, J. R., Agrawal-Gamse, C. \& Bates, P. Tetherin-mediated restriction of filovirus budding is antagonized by the Ebola glycoprotein. Proc. Natl Acad. Sci. USA 106, 2886-2891 (2009).

80. Vande Burgt, N. H., Kaletsky, R. L. \& Bates, P. Requirements within the Ebola viral glycoprotein for tetherin antagonism. Viruses 7, 5587-5602 (2015)

81. Xing, J., Ly, H. \& Liang, Y. The Z proteins of pathogenic but not nonpathogenic arenaviruses inhibit RIG-l-like receptor-dependent interferon production. J. Virol. 89, 2944-2955 (2015)

82. Hastie, K. M., King, L. B., Zandonatti, M. A. \& Saphire, E. O. Structural basis for the dsRNA specificity of the Lassa virus NP exonuclease. PLoS ONE 7, e44211 (2012).

83. Jiang, X. et al. Structures of arenaviral nucleoproteins with triphosphate dsRNA reveal a unique mechanism of immune suppression. J. Biol. Chem. 288, 16949-16959 (2013).

84. Pythoud, C. et al. Arenavirus nucleoprotein targets interferon regulatory factor-activating kinase IKK $\varepsilon$. J. Virol. 86, 7728-7738 (2012)

85. Rodrigo, W. W. et al. Arenavirus nucleoproteins prevent activation of nuclear factor kappa B. J. Virol. 86, 8185-8197 (2012).

86. Shimojima, M., Stroher, U., Ebihara, H., Feldmann, H. \& Kawaoka, Y. Identification of cell surface molecules involved in dystroglycan-independent Lassa virus cell entry. J. Virol. 86, 2067-2078 (2012)

87. Geisbert, T. W. et al. Apoptosis induced in vitro and in vivo during infection by Ebola and Marburg viruses. Lab. Invest. 80, 171-186 (2000)

This paper describes the large-scale bystander apoptosis of lymphocytes that is seen during EBOV infection.

88. Reed, D. S., Hensley, L. E., Geisbert, J. B., Jahrling, P. B. \& Geisbert, T. W. Depletion of peripheral blood T lymphocytes and NK cells during the course of Ebola hemorrhagic fever in cynomolgus macaques. Viral Immunol. 17, 390-400 (2004)
89. Wauquier, N., Becquart, P., Padilla, C., Baize, S. \& Leroy, E. M. Human fatal zaire ebola virus infection is associated with an aberrant innate immunity and with massive lymphocyte apoptosis. PLoS Negl. Trop. Dis. 4, e837 (2010)

90. Paulsen, M. \& Janssen, O. Pro- and anti-apoptotic CD95 signaling in T cells. Cell. Commun. Signal. 9, 7 (2011)

91. McElroy, A. K. et al. Human Ebola virus infection results in substantial immune activation. Proc. Natl Acad. Sci. USA 112, 4719-4724 (2015).

This study of patients with EVD treated at Emory Hospital shows that the immune response is strongly stimulated in these survivors.

92. Baize, S. et al. Defective humoral responses and extensive intravascular apoptosis are associated with fatal outcome in Ebola virus-infected patients Nat. Med. 5, 423-426 (1999)

93. Baize, S. et al. Inflammatory responses in Ebola virusinfected patients. Clin. Exp. Immunol. 128, 163-168 (2002).

This paper uses human sera from EBOV-infected patients to measure immune markers, and shows that strong anti-inflammatory responses correlate with poor outcome.

94. Schroder, K., Hertzog, P. J., Ravasi, T. \& Hume, D. A. Interferon-gamma: an overview of signals, mechanisms and functions. J. Leukoc. Biol. 75 163-189 (2004)

95. Villinger, F et al. Markedly elevated levels of interfero (IFN)-gamma, IFN-alpha, interleukin (IL)-2, IL-10, and tumor necrosis factor- $\alpha$ associated with fatal Ebola virus infection. J. Infect. Dis. 179 (Suppl. 1), S188-S191 (1999).

96. Hutchinson, K. L. \& Rollin, P. E. Cytokine and chemokine expression in humans infected with Sudan Ebola virus. J. Infect. Dis. 196 (Suppl. 2), S357-S363 (2007).

97. Couper, K. N., Blount, D. G. \& Riley, E. M. IL-10: the master regulator of immunity to infection. J. Immunol. 180, 5771-5777 (2008)

98. Fiorentino, D. F., Bond, M. W. \& Mosmann, T. R. Two types of mouse T helper cell. IV. Th2 clones secrete a factor that inhibits cytokine production by Th1 clones. J. Exp. Med. 170, 2081-2095 (1989).

99. Ouyang, J. et al. NRAV, a long noncoding RNA modulates antiviral responses through suppression of interferon-stimulated gene transcription. Cell Host Microbe 16, 616-626 (2014).

100. Panchal, R. G. et al. Induced IL-10 splice altering approach to antiviral drug discovery. Nucleic Acid. Ther 24, 179-185 (2014)

101. Leroy, E. M., Baize, S., Debre, P., Lansoud-Soukate, J. \& Mavoungou, E. Early immune responses accompanying human asymptomatic Ebola infections. Clin. Exp. Immunol. 124, 453-460 (2001). This study demonstrates that an early pro- and anti-inflammatory immune response to EBOV is protective in humans.

102. Arend W. P. The balance between IL-1 and IL-1Ra in disease. Cytokine Growth Factor Rev. 13, 323-340 (2002).

103. Ruibal, P. et al. Unique human immune signature of Ebola virus disease in Guinea. Nature 533, 100-104 (2016)

This study is the largest comprehensive examination of the human T cell response to EBOV and shows that inhibitory molecules on both $\mathrm{CD} 4^{+}$ and $\mathrm{CD}^{+} \mathrm{T}$ cells are significantly upregulated in fatal cases of EVD.

104. Gunther, S. et al. Antibodies to Lassa virus Z protein and nucleoprotein co-occur in human sera from Lassa fever endemic regions. Med. Microbiol. Immunol. 189 225-229 (2001)

105. Geisbert, T. W. et al. Development of a new vaccine for the prevention of Lassa fever. PLoS Med. 2, e183 (2005).

106. Zhou, X., Ramachandran, S., Mann, M. \& Popkin, D. L. Role of lymphocytic choriomeningitis virus (LCMV) in understanding viral immunology: past, present and future. Viruses 4, 2650-2669 (2012).

107. Meulen, J. et al. Old and New World arenaviruses share a highly conserved epitope in the fusion domain of the glycoprotein 2, which is recognized by Lassa virus-specific human CD4 + T-cell clones. Virology 321, 134-143 (2004).

108. ter Meulen, J. et al. Characterization of human CD4 T-cell clones recognizing conserved and variable epitopes of the Lassa virus nucleoprotein. J. Virol. 74 2186-2192 (2000) 
109. Pannetier, D et al. Human dendritic cells infected with the nonpathogenic Mopeia virus induce stronger T-cell responses than those infected with Lassa virus. J. Virol. 85, 8293-8306 (2011)

This paper demonstrated that highly related viruses can differentially augment dendritic cell activation and maturation, and that infection by the pathogenic, but not non-pathogenic, arenaviruses results in inhibition of dendritic cell responses.

110. Kiley, M. P., Lange, J. V. \& Johnson, K. M. Protection of rhesus monkeys from Lassa virus by immunisation with closely related Arenavirus. Lancet 2, 738 (1979).

111. Jahrling, P. B. et al. Passive immunization of Ebola virus-infected cynomolgus monkeys with immunoglobulin from hyperimmune horses. Arch. Virol. Suppl. 11, 135-140 (1996).

112. Jahrling, P. B. et al. Evaluation of immune globulin and recombinant interferon-alpha2 $b$ for treatment of experimental Ebola virus infections. J. Infect. Dis. 179 (Suppl. 1), S224-S234 (1999).

113. Marzi, A. et al. Protective efficacy of neutralizing monoclonal antibodies in a nonhuman primate mode of Ebola hemorrhagic fever. PLoS ONE 7, e36192 (2012).

114. Mupapa, K. et al. Treatment of Ebola hemorrhagic fever with blood transfusions from convalescent patients. J. Infect. Dis. 179 (Suppl. 1), S18-S23 (1999).

115. van Griensven, J. et al. Evaluation of convalescent plasma for Ebola virus disease in Guinea. N. Engl. J. Med. 374, 33-42 (2016).

116. Oswald, W. B. et al. Neutralizing antibody fails to impact the course of Ebola virus infection in monkeys. PLoS Pathog. 3, e9 (2007).

117. Parren, P. W., Geisbert, T. W., Maruyama, T. Jahrling, P. B. \& Burton, D. R. Pre- and postexposure prophylaxis of Ebola virus infection in an animal mode by passive transfer of a neutralizing human antibody. J. Virol. 76, 6408-6412 (2002).

118. Olinger, G. G. Jr et al. Delayed treatment of Ebola virus infection with plant-derived monoclonal antibodies provides protection in rhesus macaques. Proc. Natl Acad. Sci. USA 109, 18030-18035 (2012).

119. Pettitt, J. et al. Therapeutic intervention of Ebola virus infection in rhesus macaques with the MB-003 monoclonal antibody cocktail. Sci. Trans/ Med. 5, 199ra113 (2013)

120. Lyon, G. M. et al. Clinical care of two patients with Ebola virus disease in the United States. N. Engl. J. Med. 371, 2402-2409 (2014).

121. Corti, D. et al. Protective monotherapy against lethal Ebola virus infection by a potently neutralizing antibody. Science 351, 1339-1342 (2016).

122. Leroy, E. M. et al. Human asymptomatic Ebola infection and strong inflammatory response. Lancet $355,2210-2215$ (2000)

123. Marzi, A. et al. Antibodies are necessary for rVSV/ ZEBOV-GP-mediated protection against lethal Ebola virus challenge in nonhuman primates. Proc. Natl Acad. Sci. USA 110, 1893-1898 (2013). This manuscript demonstrates that antibodies elicited by a vaccine are necessary and sufficient for protection from EVD.

124. Johnson, K. M. et al. Clinical virology of Lassa fever in hospitalized patients. J. Infect. Dis. 155, 456-464 (1987).

125. Robinson, J. E. et al. Most neutralizing human monoclonal antibodies target novel epitopes requiring both Lassa virus glycoprotein subunits. Nat. Commun. 7, 11544 (2016).

126. Schountz, T. et al. Regulatory T cell-like responses in deer mice persistently infected with Sin Nombre virus. Proc. Natl Acad. Sci. USA 104, 15496-15501 (2007).

127. Basler, C. F. Innate immune evasion by filoviruses. Virology 479-480, 122-130 (2015)

128. Hoenen, T. \& Feldmann, H. Reverse genetics systems as tools for the development of novel therapies against filoviruses. Expert Rev. Anti Infect. Ther. 12, 1253-1263 (2014)

129. Martins, K. A., Jahrling, P. B., Bavari, S. \& Kuhn, J. H. Ebola virus disease candidate vaccines under evaluation in clinical trials. Expert Rev. Vaccines 15, 1101-1112 (2016).

130. Marzi, A., Feldmann, F., Geisbert, T. W., Feldmann, H. \& Safronetz, D. Vesicular stomatitis virus-based vaccines against Lassa and Ebola viruses. Emerg. Infect. Dis. 21, 305-307 (2015).

131. Mire, C. E., Geisbert, T. W., Feldmann, H. \& Marzi, A Ebola virus vaccines - reality or fiction? Expert Rev. Vaccines 15, 1421-1430 (2016).

132. Stanley, D. A. et al. Chimpanzee adenovirus vaccine generates acute and durable protective immunity against ebolavirus challenge. Nat. Med. 20 , 1126-1129 (2014).

133. De Santis, O. et al. Safety and immunogenicity of a chimpanzee adenovirus-vectored Ebola vaccine in healthy adults: a randomised, double-blind, placebo-controlled, dose-finding, phase $1 / 2$ a study. Lancet Infect. Dis. 16, 311-320 (2016).

134. Ledgerwood, J. E., Sullivan, N. J. \& Graham, B. S Chimpanzee adenovirus vector Ebola vaccine preliminary report. N. Engl. J. Med. 373, 776 (2015).

135. Jones, S. M. et al. Live attenuated recombinant vaccine protects nonhuman primates against Ebola and Marburg viruses. Nat. Med. 11, 786-790 (2005).

136. Marzi, A. et al. Ebola vaccine. VSV-EBOV rapidly protects macaques against infection with the 2014/15 Ebola virus outbreak strain. Science 349, 739-742 (2015).

137. Safronetz, D et al. A recombinant vesicular stomatitis virus-based Lassa fever vaccine protects guinea pigs and macaques against challenge with geographically and genetically distinct Lassa viruses. PLoS Negl. Trop. Dis. 9, e0003736 (2015).

138. Lukashevich, I. S. et al. A live attenuated vaccine for Lassa fever made by reassortment of Lassa and Mopeia viruses. J. Virol. 79, 13934-13942 (2005).

139. Lukashevich, I. S. Advanced vaccine candidates for Lassa fever. Viruses 4, 2514-2557 (2012).

140. Zapata, J. C. et al. An attenuated Lassa vaccine in SIVinfected rhesus macaques does not persist or cause arenavirus disease but does elicit Lassa virus-specific immunity. Virol. J. 10, 52 (2013)

\section{Acknowledgements}

This work was supported by the Division of Intramural Research, National Institutes of Allergy and Infectious Diseases, US National Institutes of Health.

Competing interests statement

The authors declare no competing interests.

\section{SUPPLEMENTARY INFORMATION}

See online article: $\mathrm{S} 1$ (table)

ALL LINKS ARE ACTIVE IN THE ONLINE PDF 